\title{
La ansiedad en voleibolistas universitarios. Análisis de la competencia deportiva en la educación superior
}

\author{
Jaime Alberto Arenas, ${ }^{1}$ Vanesa Castellanos Loaiza ${ }^{2}$ \\ Universidad del Quindío (Colombia) \\ Héctor Haney Aguirre-Loaiza, ${ }^{3}$ Catalina Trujillo ${ }^{4}$ \\ Universidad del Valle (Colombia) \\ César Núñez ${ }^{5}$ \\ Universidad de Medellín (Colombia)
}

Recibido: julio 19 de 2016. Revisado: julio 25 de 2016. Aceptado: agosto 8 de 2016

Referencia formato APA: Arenas, J., Castellanos, V., Aguirre-Loaiza, H., Trujillo, C., \& Núńez, A. (2016). La ansiedad en voleibolistas universitarios. Análisis de la competencia deportiva en la educación superior. Rev. Guillermo de Ockham, 14(2), 111-121. doi: http://dx.doi. org/10.21500/22563202.2612

\section{Resumen}

La competencia deportiva universitaria supone un contexto formativo y socializador, así como un amenazador multifactorial que exige recursos físicos y psicológicos para un rendimiento deportivo óptimo. En un estudio no experimental, de corte transversal, en una muestra de 175 voleibolistas universitarios entre diecisiete y veintiocho años $\left(M_{\text {edad }}=20,4 ; D E=2,3\right)$, se comparan los niveles de la ansiedad-estado (AE) con las muestras normales (baremos), y se estudia su variabilidad en función del carácter institucional (público frente a privado), sexo y estrato socioeconómico (bajo, medio y alto). La AE fue evaluada mediante el IDARE (STAI) previamente a la primera fase de competencia. Los resultados muestran que la $\mathrm{AE}$ varía en función del sexo y los puntajes $\mathrm{T}$ lineales $(>60)$ mostraron niveles más altos en comparación con las muestras normales (baremos). Se destacan algunas implicaciones educativas y formativas de los hallazgos y se alienta para llevar a cabo futuros trabajos en esta línea.

Palabras clave: Ansiedad, rendimiento, estudiantes universitarios, psicología aplicada, deporte

1. Magíster en Neuropsicología de la Universidad de Salamanca. Psicólogo. Licenciado en Educación Física. Docente-investigador de la Universidad del Quindío. Correo electrónico: jaarenas@uniquindio.edu.co

2. Estudiante de Licenciatura en Educación Física y Deportes de la Universidad del Quindío. vanee625@hotmail.com

3. Magíster (c) en Psicología del Instituto de Psicología de la Universidad de Valle. Psicólogo. Licenciado en Educación Física. Docente de la Universidad del Quindío. Correo electrónico: haney.aguirre@gmail.com; hector.aguirre@correounivalle.edu.co

4. Magíster (c) en Psicología del Instituto de Psicología de la Universidad del Valle. Correo electrónico: catalinatrujillo.llano@gmail.com

5. Psicólogo y magíster de la Universidad de Manizales. Doctorado en Psicología de la Universidad de Palermo, Buenos Aires, Argentina. Investigador de la Universidad de Medellín, Colombia. Miembro de la Red Global de Prácticas Clínicas, Department of Mental Health and Substance Abuse (MSD/MER), World Health Organization (OMS). Email: cnunez@udem.edu.co

Correspondencia: Instituto de Psicología Universidad del Valle. Ciudad Universitaria Meléndez. Calle 13 No 100-00. Edificio 388, Oficina 4040. Santiago de Cali, Colombia. 


\section{Anxiety in university volleyball players: Analysis of competitive sport in higher education}

\section{Abstract}

The university sport competition is a formative and socializing setting; as well as a multifactorial forbidding event, that requires physical and psychological resources for optimal athletic performance. In a non-experimental and crosssectional study, in a sample of 175 college athletes aged from 17 to 28 years $\left(M_{\text {age }}=20.4 ; \mathrm{SD}=2.3\right)$, we compare the anxiety-state (AS) levels with the normal samples (scales), and study the variability of anxiety-state (AS) according to the institutional character (public vs. private), gender and socioeconomic status (low, medium and high). The AS was evaluated by the IDARE (STAI) before the first phase of competition. The results show that the AS varies according to the gender, and linear T scores (> 60) indicated higher levels compared to normal samples (scales). We highlight the educational and formative implications of the findings and encourage further work in this line.

Keywords: Anxiety, performance, college students, applied psychology, sport

\section{A Ansiedade em jogadores de voleibol universitarios. Análise da competição desportiva no ensino superior}

\section{Resumo}

A competição deportiva universitaria requer um contexto de formação e socialização, assim como um ameaçador multifactorial que exige meios físicos e psicologicos para um desempenho deportivo ótimo. Em um estudo não experimental, de corte transversal, en uma amostra de 175 jodores de voleibol universitarios entre dezessete y vinte e oito (Medade=0,24; $\mathrm{DE}=2,3$ ), se comparam os níveles da ansiedade-estado (AE) com as amostras normais (baremos), e se estuda sua variabilidade em função do caráter institucional (público frente a privado), sexo estrato socioeconómico (baixo, meio, alto). A AE foi avaliou através el IDARE (STAI) previamente á primeira fase da competição. Os resultados mostram que a AE varia em função do sexo e os pontuações mais altos em comparação com as amostras normais (baremos). Destaca-se algumas implicaçóes educativas e anima-se para levar a cabo futuros trabalhos em esta linha.

Palavras-chave: Ansiedade, desempenho, estudantes, universitarios, psicologia aplicada, deporte

\section{Introducción}

Las universidades son un escenario diverso y socializador cuyo fin es la formación de ciudadanos fundamentados en pilares misionales como generación de conocimiento, extensión y proyección social. El rendimiento académico es el principal marcador de éxito de los estudiantes, y este parece estar determinado por factores personales, familiares y situacionales (Khalaila, 2015). La presentación de pruebas académicas constituye un factor situacional que demanda recursos psicológicos y físicos para que dicho rendimiento sea óptimo. Asimismo, la ansiedad es un factor personal que se ha relacionado con el rendimiento académico (Chapell et al., 2005; Farooqi, Ghani, \& Spielberger, 2012). Se ha reportado que algunas técnicas terapéuticas son alternativas para enfrentar estas demandas (Ahmed, 2015), entre ellas, el ejercicio físico sistemático confiere efectos benéficos ante las exigencias académicas (Von Haaren, Haertel, Stumpp, Hey, \& Ebner-Priemer, 2015).

Adicionalmente, el rendimiento académico tiene implicaciones indirectas en actividades extracurriculares; por ejemplo, constituye un criterio para la selección e integración de equipos y delegaciones deportivas y con ello, la posibilidad de conseguir reconocimientos personales y sociales (Gayles, 2009). Se sabe que los estudiantes deportistas invierten gran parte de su tiempo en los entrenamientos deportivos, experimentan fatiga o lesiones, y pierden numerosas clases durante las salidas a la competencia (Watt \& Moore, 2001); y aún así, deben mantener un buen rendimiento académico con el fin de conservar su elegibilidad en estas delegaciones (Gayles, 2009). Así, el estudiante deportista combina su desempeño académico y deportivo, lo que al parecer genera un efecto positivo sobre sí mismo y la universidad (Gayles 
\& $\mathrm{Hu}, 2009)$. Igualmente, tras la consecución de logros deportivos, las universidades refuerzan al deportista mediante becas, incentivos y créditos educativos (Weinberg \& Gould, 2010).

Según lo anterior, la competencia deportiva y sus logros son factores motivacionales mediante los cuales los estudiantes buscan estímulos o reforzadores positivos. Por tal razón, esta fase es una condición situacional similar al examen académico que puede generar altos niveles de ansiedad. Al respecto, los estudios se han enmarcado en dos líneas: 1. la identificación de factores potenciales en la génesis de ansiedad durante la competencia deportiva; y 2. la capacidad de repuesta frente a ella en dicha fase (Mellalieu, Hanton \& Fletcher, 2006). En la primera, se han señalado la preparación física y mental, la naturaleza del evento y la evaluación social y personal (Hanton, Neil, \& Mellalieu, 2008) como fuentes potenciales de tensión para el estudiante atleta. Así, se ha indicado que evaluaciones psicológicas previas a la competencia evidencian mayores puntuaciones en ansiedad cognitiva, y al momento de llevarse a cabo mayores puntuaciones en ansiedad somática y autoconfianza (Prieto, 2016). En esta línea se centra el estudio.

Según la evaluación cognitiva que el deportista hace del ambiente, se puede generar un estado emocional transitorio que se caracteriza por sentimientos de tensión y aprensión percibidos conscientemente y un incremento de la actividad del sistema nervioso autónomo, que se manifiesta mediante señales fisiológicas como incremento de la frecuencia cardíaca, dilatación de la pupila y sudoración (Mannino \& Robazza, 2004), fenómeno conocido en la literatura como ansiedad-estado (AE). Por otro lado, puede generarse la ansiedad-rasgo, entendida como una disposición conductual inherente a la personalidad que hace que situaciones no peligrosas se perciban como amenazantes y se responda a ellas en ese sentido (Spielberger, Gorsuch, \& Lushene, 1970). Para los propósitos de este estudio nos enfocaremos en la $\mathrm{AE}$.

En esta línea, la competencia deportiva es un disparador de la AE (Hanton et al., 2008; Mellalieu et al., 2006), ya que además de dirigirse a la consecución de reforzadores (becas, incentivos, créditos), se ponen a prueba los límites físicos y psicosociales del sujeto y por consiguiente, la ejecución deportiva (Landers \& Boutcher, 1991). Si bien la $\mathrm{AE}$ en fases precompetitivas puede ser facilitadora del rendimiento deportivo (Wiggins \& Brustad, 1996) también puede afectarlo negativamente (Abenza, Alarcon, Leite, Urena, \& Pinar, 2009)”. Abenza, Alarcon, Leite, Urena, \& Pinar, 2009. En resumen, las evaluación del entorno, los mecanismos de afrontamiento y las experiencias individuales parecen regular los niveles de ansiedad. Igualmente, llevar a cabo nuevas investigaciones es una necesidad detectada en el marco de nuestra línea de trabajo (Aguirre-Loaiza \& Gonzalez, 2014; Aguirre-Loaiza \& Ramos, 2011; Arenas \& Aguirre-Loaiza, 2014).

Algunos de nuestros estudios han aportado a la descripción y relación de las variables psicológicas en contextos deportivos. Así, hemos reportado que la AE incrementa paralelamente con el avance de las fases competitivas (Arenas \& Aguirre-Loaiza, 2014), sus puntajes son altos según la posición de juego en futbolistas juveniles (Aguirre-Loaiza \& Ramos, 2011, 2013) aunque sin mostrar diferencias significativas (Fernández et al., 2014, AguirreLoaiza \& Ramos, 2011), y que esta se diferencia según las condiciones sociodemográficas y la experiencia deportiva (Aguirre-Loaiza \& Ramos, 2011). Asimismo, hemos señalado que la combinación de factores motivacionales extrínsecos (refuerzos al conseguir un logro) e intrínsecos (placer por el aprendizaje y auto superación) caracterizan el perfil motivacional de deportistas universitarios, el cual parece asociarse con distintos niveles de ansiedad (AguirreLoaiza \& Gonzalez, 2014). Finalmente, hemos evidenciado que las características psicológicas varían según la modalidad deportiva y el sexo en deportes de conjunto (Arias, Cardozo, Aguirre-Loaiza, \& Arenas, 2016).

De lo anterior se infiere que la ansiedad podría estar afectada por los recursos personales, el tipo de modalidad deportiva y las condiciones escolares (combinación de actividades escolares y extracurriculares, rendimiento académico y deportivo, búsqueda de incentivos, etc.). Además, por las condiciones deportivas principalmente la competencia, entendida como un elemento socializador y mediador entre los procesos psicológicos y deportivos, que en esencia son formadores. Esta y sus implicaciones en el deporte universitario, como mantener un buen rendimiento académico con el fin de integrar los equipos y delegaciones deportivas, tiene cuestiones sin resolver, como la comparación de los puntajes de AE con muestras normativas y estudiar si sus niveles difieren según el carácter institucional (público frente a privado), el sexo y el estrato socioeconómico. En este sentido, nuestra hipótesis sostiene que los estudiantes universitarios evalúan la competencia deportiva como un ambiente amenazador multifactorial que exige recursos psicológicos y físicos que permitan mantener o mejorar un rendimiento deportivo esperado general, altos niveles de $\mathrm{AE}$ y, además, están acompañadas de características personales y sociales que pueden variar, como el carácter de la institución universitaria, el sexo, y el estrato socioeconómico. 
Por lo tanto, este estudio pretende: 1 . identificar si hay diferencias en los niveles de $\mathrm{AE}$ entre los deportistas universitarios, las muestras normales (baremos) y muestras de otros deportistas (fútbol y voleibol), y 2. estudiar el comportamiento de la $\mathrm{AE}$ en relación con las universidades públicas y privadas, el sexo y el estrato socioeconómico en la práctica deportiva (competencia).

\section{Método}

\section{Diseño y participantes}

Estudio no experimental, de alcance descriptivo y corte transversal (Kerlinger, 1988). Mediante un muestreo por censo, se evaluaron 175 voleibolistas universitarios, conformados por noventa mujeres $(51,4 \%)$ y 85 hombres $(48,6 \%)$, con un rango de edad entre los diecisiete y los veintiocho 28 ( $\left.M_{\text {edad }}=20.4 ; D E=2,3\right)$, experiencia deportiva entre uno y cinco años $(M=2,9 ; D E=1,3)$, y provenientes de los seis estratos socioeconómicos categorizados de la siguiente manera: bajo (estratos 1 y 2), medio (estratos 3 y 4), y alto (estratos 5 y 6). Los participantes representaban diez instituciones de educación superior del centrooccidente de Colombia, cinco públicas (Universidad de Caldas; Universidad Tecnológica del Chocó; Universidad Nacional de Colombia, sede Manizales; Universidad Tecnológica de Pereira, y Universidad del Quindío), y cinco privadas (Fundación Universitaria del Área Andina, sede Pereira; Universidad Católica de Manizales; Universidad La Gran Colombia, sede Armenia; Universidad Libre, sede Pereira, y Universidad de Manizales). Los criterios de inclusión fueron ser universitario y estar inscrito en competencia de cada delegación universitaria.

\section{Instrumentos}

Se administró un cuestionario de autoinforme ad hoc para la medición de las variables sociodemográficas como el sexo, la edad, y el estrato socioeconómico (ESE) (bajo, medio y alto); la institución educativa determinó el carácter público o privado.

La ansiedad-estado se evaluó mediante el State-Tait Anxiety Inventory (STAI) (Spielberger et al., 1970), usando la versión en castellano Inventario de ansiedad rasgo-estado (Idare) (Spielberger \& Guerrero-Díaz, 2002). Este instrumento está compuesto por dos escalas: ansiedad-rasgo y ansiedad-estado. Esta última, de interés del presente estudio, consta de veinte reactivos, medidos de manera indirecta $(1,2,5,8,10,11,15,16,19$ y 20$)$ y los restantes formulados de forma directa. La puntuación es tipo Likert, así: 1: "no en absoluto"; 2: "un poco"; 3: "bastante", y 4: "mucho". El puntaje bruto se transformó a una escala normalizada de puntuación $\mathrm{T}$, categorizada en baja (menor a 40), media (entre 40 y 60 ) y alta (mayor a 61). Aunque este instrumento no consta de criterios específicos aplicados a la psicología del deporte, su validez y utilidad en este campo han sido demostradas (Dosil, 2008; Guillén-Riquelme \& Buela-Casal, 2011; Roffé, 2009). Psychometric revision and differential item functioning in the State Trait Anxiety Inventory (STAI y es útil a la hora de evaluar la ansiedad en el deporte (Mellalieu et al., 2006). En el presente estudio se estimó un valor de fiabilidad de $\alpha=0.8$, calculado mediante el método de consistencia interna.

\section{Procedimiento y consideraciones éticas}

La recolección de los datos se llevó a cabo en el Coliseo Menor de la Ciudad de Manizales, Colombia, durante el desarrollo del zonal universitario organizado por la Asociación Colombiana de Universidades (Ascún), efectuado del 27 al 30 de agosto de 2015. Los equipos que compitieron en el zonal eran procedentes de la región centro-occidente del país. Los estudiantes accedieron voluntariamente a participar de la investigación. En la primera fase del zonal universitario se evaluó la $\mathrm{AE}$ una hora antes de cada encuentro, previa autorización del cuerpo técnico de cada delegación universitaria. Luego de la aprobación, se continuó con la aplicación de la escala de $\mathrm{AE}$ con base en la información escrita y verbal de los diferentes apartados del consentimiento informado (aspectos éticos). Los datos fueron recolectados por un psicólogo y dos estudiantes de educación física y deportes de último año, quienes fueron entrenados en la aplicación del consentimiento, el autoinforme ad hoc y el inventario de AE.

Para cumplir lo dispuesto en la Resolución 8430, en los lineamientos de la Ley 1090 y en Código Deontológico y Bioético del Ejercicio de la Psicología en Colombia (Congreso de la Republica de Colombia, 2006) y antes de la evaluación, los participantes firmaron el consentimiento informado en el cual se les garantizó la confidencialidad, la posibilidad de reiterarse en cualquier momento y el uso de los datos únicamente para los propósitos del estudio.

\section{Análisis de datos}

Mediante un análisis estadístico univariado y bivariado y el uso del paquete estadístico SPSS v.20, se dio cumplimiento a lo objetivos. Para el análisis bivariado de las variables nominales se empleó el análisis de frecuencias de tablas de contingencias con el estadístico de chi cuadrado $\left(x^{2}\right)$, cruzando niveles de ansiedad (medio-alto) por: carácter 
institucional (público-privado), sexo (hombres-mujeres) y ESE (bajo, medio y alto). El análisis univariado se calculó por medio del estadístico de $t$ de student para una muestra en la que se compararon las medias. El tamaño del efecto d de Cohen se estableció mediante $G^{*}$ Power (Faul, Erdfeld, Lang, \& Buchner, 2007; Faul, Erdfelder, Buchner, \& Lang, 2009) y los parámetros interpretativos fueron: 0,20: pequeño; 0,50: mediano, y 0,80: grande (Cohen, 1988; Ferguson, 2009). El coeficiente de confiabilidad de las medidas del STAI para este estudio se calculó con el alfa de Cronbach. Se asumió un nivel de significancia menor a $0.05(p<0.05)$.

\section{Resultados}

\section{Ansiedad-estado por universidades}

Los datos descriptivos de frecuencias y comportamiento de la $\mathrm{AE}$ en función a las universidades participantes se describen la Tabla 1 y en la Figura 1. La mayoría de los estudiantes pertenecen a universidades del Eje Cafetero y están distribuidos de la siguiente manera: universidades con sede en Manizales (Caldas, Católica, Manizales y Nacional, 40,6\%); en Pereira (Área Andina, Libre y Tecnológica, 29,7 \%), en Armenia (Gran Colombia y Quindío, 17,2 \%) y en Quibdó (Tecnológica de Chocó, 12,6 \%). La distribución de instituciones públicas $(n=105,60 \%)$ fue superior en comparación a las privadas $(n=70,40 \%)$.

Tabla 1

Distribución de frecuencias y puntajes de ansiedad-estado por delegación universitaria

\begin{tabular}{lccc}
\hline \multirow{2}{*}{ Institución universitaria } & \multicolumn{3}{c}{ Ansiedad-estado } \\
\cline { 2 - 4 } & $\mathbf{n}(\%)$ & $\mathbf{M}$ & $\mathbf{D E}$ \\
\hline 1. Área andina & $19(10,9)$ & 60,7 & 3,8 \\
\hline 2. Caldas & $14(8,0)$ & 63,1 & 5,1 \\
\hline 3. Católica de Manizales & $15(8,6)$ & 61,3 & 4,2 \\
\hline 4. Tecnológica de Chocó & $22(12,6)$ & 62,5 & 3,2 \\
\hline 5. Gran Colombia & $8(4,6)$ & 64,2 & 4,4 \\
\hline 6. Libre & $9(5,1)$ & 63,3 & 5,3 \\
\hline 7. Manizales & $19(10,9)$ & 61,1 & 3,2 \\
\hline 8. Nacional (sede Manizales) & $23(13,1)$ & 60,3 & 3,5 \\
\hline 9. Tecnológica de Pereira & $24(13,7)$ & 62,5 & 3,2 \\
\hline 10. Quindío & $22(12,6)$ & 61,4 & 2,4 \\
\hline Total & $175(100,0)$ & 61,8 & 3,7 \\
\hline
\end{tabular}

Fuente: Elaboración propia.

Según los puntajes $\mathrm{T}$, los deportistas universitarios se encuentran en niveles altos de $\mathrm{AE}$ comparados con los datos normativos de una población general. La Figura 1 traza el comportamiento de la $\mathrm{AE}$ antes de la primera ronda de la competencia deportiva. Los valores de $60 \mathrm{y}$ superiores indican altos niveles $\mathrm{AE}$.
Figura 1

Puntajes T de ansiedad-estado antes de la competencia deportiva en universitarios.



Fuente: Elaboración propia.

Nota: 1 = Área Andina; 2 = Caldas; 3 = Católica de Manizales; 4 =

Tecnológica de Chocó; 5 = Gran Colombia; 6 = Libre; 7 = Manizales; $8=$ Nacional (sede Manizales) ; $9=$ Tecnológica de Pereira; $10=$ Quindío.

\section{Niveles de ansiedad-estado según el carácter institucional, el sexo y el estrato socioeconómico}

El estudio de las posibles diferencias de la $\mathrm{AE}$ en función del carácter institucional, el sexo y el estrato socioeconómico se hizo mediante un análisis de chi cuadrado en los niveles de AE "medio" y "alto" (Tabla 2). No se reportaron frecuencias en el nivel "bajo" de la $\mathrm{AE}$.

Tabla 2

Chi cuadrado según el carácter institucional, el sexo y el estrato socioeconómico

\begin{tabular}{|c|c|c|c|c|}
\hline \multirow[t]{2}{*}{ Variables } & \multicolumn{2}{|c|}{ Nivel de ansiedad } & \multirow[b]{2}{*}{$\mathrm{X}^{2}$} & \multirow[b]{2}{*}{$p$} \\
\hline & $\begin{array}{c}\text { Medio } \\
\mathbf{n}(\%)\end{array}$ & $\begin{array}{l}\text { Alto } \\
\text { n(\%) }\end{array}$ & & \\
\hline \multicolumn{5}{|c|}{ Carácter institucional } \\
\hline Pública & $26(54,2)$ & $79(62,2)$ & \multirow{2}{*}{0,93} & \multirow{2}{*}{0,33} \\
\hline Privada & $22(45,8)$ & $48(37,8)$ & & \\
\hline \multicolumn{5}{|l|}{ Sexo } \\
\hline Mujeres & $31(64,6)$ & $59(46,5)$ & \multirow{2}{*}{4,58} & \multirow{2}{*}{0,03} \\
\hline Varones & $17(35,4)$ & $68(53,5)$ & & \\
\hline \multicolumn{5}{|l|}{ ESE } \\
\hline Bajo & $10(20,3)$ & $53(41,7)$ & \multirow{3}{*}{8,32} & \multirow{3}{*}{0,16} \\
\hline Medio & $33(68,8)$ & $57(44,9)$ & & \\
\hline Alto & $5(10,4)$ & $17(13,4)$ & & \\
\hline
\end{tabular}

Fuente: Elaboración propia.

Nota: $\mathrm{X}^{2}=$ chi cuadrado; $\mathrm{ESE}=$ estrato socioeconómico

De acuerdo con la Tabla 2, los estudiantes de universidades públicas, hombres y de estrato socioeconómico medio, presentaron altos niveles de $\mathrm{AE}$ previo a la competencia. Se presenta significancia estadística en el sexo $\left[\mathrm{X}^{2}=\right.$ $(1,175)=4.58, p=0.03]$, lo que indica que las frecuencias observadas entre los niveles medio y alto, $y$ hombres $y$ mujeres son diferentes. La distribución de frecuencias en cuanto al carácter institucional y el ESE es homogénea en los niveles medio y alto de la AE. La Figura 2 apoya la presentación de estos resultados. 
Figura 2

Análisis de chi cuadrado de la frecuencia de ansiedad-estado en los niveles medio y alto

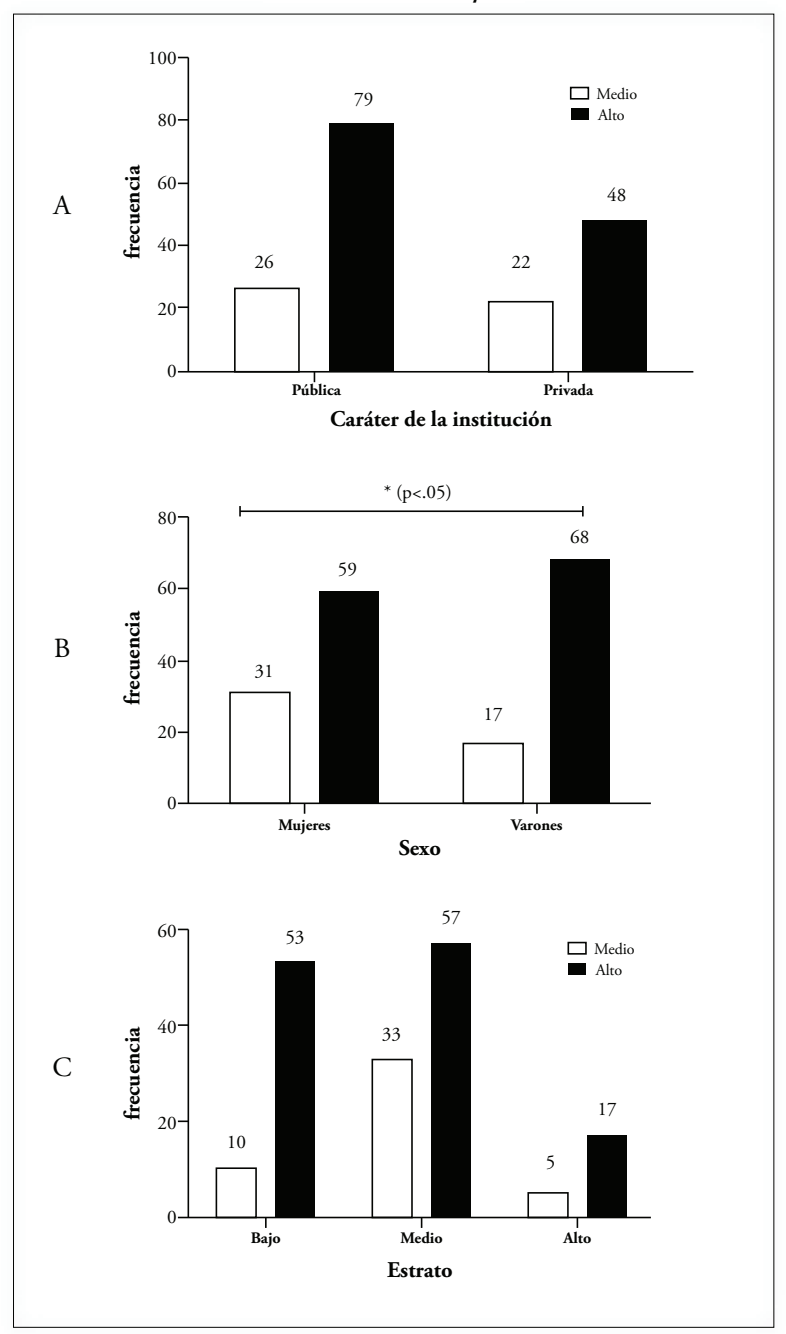

Fuente: Elaboración propia.

$\mathrm{A}=$ carácter institucional; $\mathrm{B}=$ sexo $\mathrm{C}=$ estrato socioeconómico. ${ }^{*} \mathrm{p}<0.05$

\section{Comparación con otras muestras}

La Tabla 3 muestra la comparación de la AE con los datos normativos del STAI en el grupo de "universitarios no-graduados" hombres y mujeres de la Universidad Estatal de Florida (Spielberger et al., 1970), hallándose diferencias significativas $t(175)=34.4, p=.000$. Igualmente, al contrastarse con otros trabajos cuya aplicación metodológica y condiciones de competencia han sido similares, se establecieron diferencias significativas $(p<$. $01)$. Por otro lado, en comparación con las muestras de voleibolistas universitarios y futbolistas amateurs las diferencias en el tamaño del efecto son pequeñas; mientras que en comparación a los futbolistas colombianos $(d=1,60)$ y la muestra normativa del STAI $(d=1,40)$, las diferencias en el tamaño del efecto son grandes.
Tabla 3

Comparación de la ansiedad-estado con otras muestras

\begin{tabular}{|c|c|c|c|c|c|c|}
\hline Estudios & Muestras & $\mathrm{M}(\mathrm{DE})$ & $\begin{array}{c}t \\
(174)\end{array}$ & $\mathrm{p}$ & $\begin{array}{c}95 \% \text { IC } \\
\text { LI LS }\end{array}$ & $\begin{array}{c}\text { TE } \\
\text { d }\end{array}$ \\
\hline $\begin{array}{l}\text { (Arenas \& } \\
\text { Aguirre-Loai- } \\
\text { za, 2014) }\end{array}$ & $\begin{array}{l}\text { Voleibolistas } \\
\text { universitarios } \\
(\mathrm{n}=98)\end{array}$ & $60,6(4,0)$ & 4,24 & 0,000 & {$[0,65,1,77]$} & 0,29 \\
\hline $\begin{array}{l}\text { (Aguirre- } \\
\text { Loaiza } \\
\text { \& Ramos, } \\
\text { 2013) }\end{array}$ & $\begin{array}{l}\text { Futbolistas } \\
\text { aficionados } \\
(\mathrm{n}=110)\end{array}$ & $61,0(3,9)$ & 2,84 & 0,005 & {$[0,25,1,37]$} & 0,18 \\
\hline $\begin{array}{l}\text { (Aguirre- } \\
\text { Loaiza } \\
\& \text { Ramos, } \\
\text { 2011) }\end{array}$ & $\begin{array}{l}\text { Futbolistas } \\
\text { colombianos } \\
(\mathrm{n}=93)\end{array}$ & $49,6(10,0)$ & 42,8 & 0,000 & {$[11,6,12,7]$} & 1,60 \\
\hline $\begin{array}{l}\text { (Spielberger } \\
\text { et al., 1970) }\end{array}$ & $\begin{array}{l}\text { Universita- } \\
\text { rios - Nor- } \\
\text { mas STAI } \\
(\mathrm{n}=484)\end{array}$ & $51,5(9,3)$ & 34,4 & 0,000 & {$[9,55,10,8]$} & 1,40 \\
\hline
\end{tabular}

Fuente: Elaboración propia.

Nota: los datos fueron contrastados con los estudios referenciados.

Los estadísticos son: $\mathrm{n}=175 ; \mathrm{M}=61,7 ; \mathrm{DE}=3,7$. Todos los puntajes están en una escala lineal de $\mathrm{T}=\mathrm{M}=50 ; \mathrm{DE}=10$.

$\mathrm{IC}=$ intervalo de confianza; $\mathrm{LI}=$ límite inferior; $\mathrm{LS}$ = límite superior; $\mathrm{TE}=$ tamańo del efecto.

\section{Discusión}

De acuerdo con el primer objetivo consistente en determinar si hay diferencias en los niveles de $\mathrm{AE}$ entre los deportistas universitarios y las muestras normales (baremos), se encontró que los puntajes de $\mathrm{AE}$ de los universitarios voleibolistas fueron significativamente superiores en comparación con los datos normativos de la población general de estudiantes $(<60$, nivel superior) (Spielberger et al., 1970). Por consiguiente, se confirma nuestra hipótesis de que la competencia deportiva en el ámbito universitario constituye una fuente de ansiedad. Otros trabajos que han empleado el paradigma de autorreporte de respuestas cognitivas y conductuales previo al evento competitivo, son consistentes con nuestros hallazgos al reportar altos niveles de $\mathrm{AE}$ en futbolistas aficionados antes de la fase de semifinal (Aguirre-Loaiza \& Ramos, 2013), $y$ un incremento de esta a medida que avanzan las fases de la competencia en deportistas universitarios (Arenas \& Aguirre-Loaiza, 2014).

Lo anterior puede asociarse con la mediación de factores deportivos, académicos y personales en el desempeño y la ansiedad de los participantes. En este sentido, la $\mathrm{AE}$ se ha asociado con algunas características deportivas, como la posición de juego (Aguirre-Loaiza \& Ramos, 2011, 2013), la experiencia deportiva (Aguirre-Loaiza \& Ramos, 2011) y la fase competitiva (Arenas \& AguirreLoaiza, 2014). Asimismo, en los deportistas universitarios, media una serie de componentes académicos y educativos como la disponibilidad de recursos económicos para 
incentivar el deporte, el apoyo institucional tanto a los deportistas como a los programas deportivos (reflejado en becas, incentivos, créditos educativos, apoyo para que la práctica deportiva no interfiera con el rendimiento académico óptimo), la forma como se concibe el deporte (socializador o no, formador o no) (Guardia, 2004), y las posibles alteraciones del rendimiento académico debido a la práctica deportiva (Gayles, 2009). En su conjunto, estos factores parecen ser fuentes potenciales de ansiedad en los jóvenes deportistas.

Adicionalmente, algunos factores personales como la manera como se evalúa cognitivamente la competencia deportiva previa y en su transcurso y sus posibles consecuentes (Gayles \& Hu, 2009; Gayles, 2009); el nivel de habilidad percibida y los mecanismos de afrontamiento (Jones, Swain, \& Cale, 1991; Jones, 1995), y la preparación física, táctica, técnica y mental (Dosil, 2008; Weinberg \& Gould, 2010), se han asociado con la ansiedad. Igualmente, en este estudio el sexo diferenció los niveles de $\mathrm{AE}$, al encontrarse que estos son más altos en los hombres. Lo anterior apoya los trabajos previos que han reportado diferencias en función de esta variable en la competencia deportiva (Hanton et al., 2008; Jones et al., 1991; Mellalieu et al., 2006; Woodman \& Hardy, 2003). Sin embargo, contradice otros que han reportado mayor ansiedad en las mujeres (Jones et al., 1991; Woodman \& Hardy, 2003) y ninguna diferencia en relación con el sexo (Koca, 2015; Ruiz-Juan \& Zarauz, 2014).

$\mathrm{Al}$ respecto, los estudios no son concluyentes y a nuestro criterio la inconsistencia subyace a un problema metodológico ante la diversidad de instrumentos usados en las medidas del constructo de ansiedad. Sin embargo, una explicación en función de la variable de sexo como factor individual, puede ser la facilidad de las mujeres de reportar sentimientos desagradables y con menos presión por la deseabilidad social (Mellalieu et al., 2006). También se ha observado que los hombres presentan una tendencia a la competencia y a la victoria, mientras las mujeres direccionan sus conductas a objetivos (Jamshidi, Hossien, Sajadi, Safari, \& Zare, 2011), lo cual es coherente en adolescentes en ambientes escolares (Amado, Sánchez-Miguel, Leo, Sánchez-Oliva, \& García-Calvo, 2012). Así, para los hombres se espera que la dirección de tareas y metas esté en función de situaciones de recompensa (Moreno, López, Martínez, Villodre, \& Gonzallez-Cutre, 2007) y la motivación extrínseca en deportistas universitarios ha sido un perfil característico (Aguirre-Loaiza \& González, 2014).

Por otro lado, factores contextuales como el carácter universitario (público frente a privado) y el estrato so- cioeconómico no diferenciaron el comportamiento de la $\mathrm{AE}$ en los niveles medio y alto. Lo anterior dista de estudios previos que han reportado diferencias en la $\mathrm{AE}$ en función de variables sociodemográficas, como el estrato socioeconómico en deportistas universitarios (AguirreLoaiza \& Ramos, 2011). La posible variabilidad de la $\mathrm{AE}$ en relación con estas variables, suponía una posible repercusión de la disponibilidad de recursos económicos y el apoyo institucional al deporte sobre el estado psicológico de los participantes, lo cual, a su vez, podría ser modulado por habilidades psicológicas como la cohesión de equipo (Eys, Hardy, Carron, \& Beauchamp, 2003) y el sentido de pertenencia (Jones, 1995). De acuerdo con lo mencionado, el segundo objetivo consistente en estudiar el comportamiento de la $\mathrm{AE}$ en relación con las universidades públicas y privadas, el sexo y el estrato socioeconómico en la competencia, parecen marcar una tendencia en los nuevos estudios hacia las diferencias individuales (Mellalieu et al., 2006), y quizás comprenderse como posibles variables predictoras con relativo énfasis en los procesos cognitivos.

De esta manera, las experiencias deportivas, académicas y personales parecen ser factores amortiguadores en la medida en que modulan los niveles de ansiedad, permiten el despliegue de habilidades psicológicas básicas ante la aparición de sus síntomas (Wadey \& Hanton, 2008) y favorecen el manejo adecuado de situaciones exigentes al servir como indicador sensible de la habilidad psicológica (Hanton et al., 2008). Además de las ya conocidas dimensiones cognitivas y fisiológicas, se ha propuesto una tercera dimensión reguladora conocida como modelo tridimensional de medida del rendimiento de la ansiedad (Cheng, Hardy, \& Markland, 2011, 2009), que informa sobre su condición positiva y adaptativa (Cheng \& Hardy, 2016), de modo que la adaptación, el diálogo interno y las estrategias de afrontamiento pueden ser habilidades psicológicas que facilitan el desempeño (Cheng \& Hardy, 2016) y desde luego, puede ser aplicado a las experiencias académicas.

Los altos niveles de AE observados en los estudiantes universitarios deportistas señalan algunas implicaciones para las instituciones de educación superior. En este sentido, es necesario desarrollar en estas instituciones, estrategias de fomento y protección al deporte y hacer de él un entorno socializador y formativo y un elemento para mejorar la salud y la calidad de vida (Guardia, 2004). Estas estrategias deberán atraer nuevos estudiantes a la práctica deportiva y apoyar económicamente a los deportistas y a los programas deportivos mediante becas, incentivos o estímulos. Igualmente, se deberá hacer seguimiento y 
brindar atención educativa permanente a los deportistas para que el desempeño académico no se vea afectado por la práctica deportiva, así como articular las estrategias deportivas y educativas (Guardia, 2004).

Asimismo, estos programas deben fundamentarse y articularse con los hallazgos teóricos sobre los efectos benéficos potenciales del deporte en las universidades, tales como la optimización de las estrategias de afrontamiento, la autoestima y la autopercepción, la generación de recursos motivacionales, el mejoramiento del relacionamiento social (Guardia, 2004) y el rendimiento académico (Capdevila, Bellmunt, \& Domindo, 2015). Dicho sustento teórico sienta la necesidad de incluir el deporte en una concepción integral de formación, la cual, en términos específicos, no es otra cosa que la oportunidad de que las universidades combinen los programas académicos y deportivos con pedagogías inclusivas que aporten al desarrollo profesional y personal, una perspectiva sin duda coherente con los proyectos educativos institucionales $\mathrm{y}$ las perspectivas misionales y visionales de la educación superior.

Finalmente, como limitaciones del estudio y aspectos que se deben tener en cuenta en el futuro, se destaca el control de variables (respuestas fisiológicas) con medidas complementarias, lo cual podría contribuir a la comprensión del fenómeno. Asimismo, cabe considerar el nivel de rendimiento deportivo, la experiencia deportiva y el tipo de deportes, ya que estos serían predictores situacionales del comportamiento de AE. Además, contrastar paralelamente situaciones académicas y deportivas; por ejemplo, la presentación de exámenes y la competencia deportiva en los universitarios deportistas, serán de utilidad para comprender los factores contextuales o personales relacionados con la AE. De esta manera, nuevos alcances investigativos en ambientes universitarios serán propicios para nutrir el cuerpo del conocimiento, cuyas implicaciones teóricas son de provecho para el deporte universitario.

En conclusión, la competencia deportiva se evalúa como un ambiente amenazante. Los estudiantes voleibolistas presentan altos niveles $\mathrm{AE}$ en ella, con diferencias estadísticamente significativas en relación con la con la población general y otras muestras de deportistas. Respecto de las variables estudiadas, el sexo diferencia los niveles medio y alto y se reportan niveles más altos de $\mathrm{AE}$ en los hombres, mientras que el carácter institucional y el ESE no parecen diferenciar los niveles de AE. Puede pensarse que la experiencia deportiva y personal se debe considerar a la hora de diseñar estrategias de afrontamiento, así como el apoyo de profesionales en el aprendizaje de técnicas.

\section{Referencias}

Abenza, L., Alarcon, F., Leite, N., Urena, N., \& Pinar, M. (2009). Relación entre la ansiedad y la eficacia de un equipo de baloncesto durante la competicion. Cuadernos de Psicología del deporte, 9 (Supple), 51.

Aguirre-Loaiza, H. H., \& González, F. (2014). Factores motivacionales, variables sociodemográficas y deportivas en deportistas universitarios. Tesis psicológica, 9(1), 130-145.

Aguirre-Loaiza, H. H., \& Ramos, S. (2011). Ansiedad-estado y variables sociodemográficas en futbolistas juveniles colombianos durante competencia. Diversitas: Perspectivas en psicología, 7(2), 239-251.

Aguirre-Loaiza, H. H., \& Ramos, S. (2013). Ansiedad ante la competencia en futbolistas: Comparación entre titulares y suplentes. Avances de la psicología del deporte en Iberoámerica, 2(1), 47-61.

Ahmed, Z. (2015). Pre-exam anxiety among students and its coping mechanism. Rawal Medical Journal, 40(2), 233-236.

Amado, D., Sánchez-Miguel, P. A., Leo, F. M., Sánchez-Oliva, D., \& García-Calvo, T. (2012). Diferencias de género en la motivación y percepión de utilidad del deporte escolar. Revista internacional de medicina y ciencias de la actividad fisica y del deporte, 14(56), 651-664.

Arenas, J., \& Aguirre-Loaiza, H. (2014). Ansiedad-estado a lo largo de las fases de competencia deportiva. Lúdica pedagógica, 19(1), 111-121.

Arias, I. A., Cardozo, T., Aguirre-Loaiza, H. H., \& Arenas, J. (2016). Características psicológicas del rendimiento deportivo en deportes de conjunto: diferencias entre modalidad y género. Psicogente, 19(35), 25-36.

Capdevila, A., Bellmunt, H., \& Domindo, C. (2015). Estilo de vida y rendimiento académico en adolescentes: comparación entre deportistas y no-deportistas. Revista de estudios sociales, 27, 28-33.

Chapell, M. S., Benjamin Blanding, Z., Takahashi, M., Silverstein, M. E., Newman, B., Gubi, A., \& McCann, N. (2005). Test anxiety and academic performance in undergraduate and graduate students. Journal of Educational Psychology, 97(2), 268-274. http://doi.org/10.1037/00220663.97.2.268

Cheng, W.-N., \& Hardy, L. (2016). Three-dimensional model of performance anxiety: Tests of the adaptive potential of the regulatory dimension of anxiety. Psychology of Sport and Exercise, 22, 255-263. http://doi.org/10.1016/j.psychsport.2015.07.006

Cheng, W.-N., Hardy, L. E. W., \& Markland, D. (2011). Crosscultural validation of a three- dimensional measurement model of performance anxiety in the Context of Chinese 
Sports Context of Chinese Sports . International Journal of Sport Psychology, 42(5), 417-435.

Cheng, W.-N., Hardy, L., \& Markland, D. (2009). Toward a three-dimensional conceptualization of performance anxiety: Rationale and initial measurement development. Psychology of Sport and Exercise, 10(2), 271-278. http://doi. org/10.1016/j.psychsport.2008.08.001

Cohen, J. (1988). Statistical power analysis for the behavioral sciences. Statistical Power Analysis for the Behavioral Sciences. http://doi.org/10.1234/12345678

Colegio Colombiano de Psicólogos. Deontología y bioética del ejercicio de la psicología en Colombia (2012). Bogotá DC.

Congreso de la República de Colombia. Ley 1090 (2006). Bogotá DC.

Dosil, J. (2008). Psicología de la actividad fisica y el deporte (2a ed.). Madrid: McGraw-Hill.

Eys, M. A., Hardy, J., Carron, A. V., \& Beauchamp, M. R. (2003). The relationship between task cohesion and competitive state anxiety. Journal of Sport \& Exercise Psychology, 25, 66-76. Retrieved from http://hdl.handle.net/10242/41236

Farooqi, Y., Ghani, R., \& Spielberger, C. (2012). Gender Differences in Test Anxiety and Academic Performance of Medical Students. International Journal of Psychology and Behavioral Sciences, 2(2), 38-43. http://doi.org/10.5923/j. ijpbs.20120202.06

Faul, F., Erdfeld, E., Lang, A. G., \& Buchner, A. (2007). A flexible statistical power analysis program for the social, behavioral, and biomedical sciences. Behavior Research Methods, 39(2), 175-191.

Faul, F., Erdfelder, E., Buchner, A., \& Lang, A.-G. (2009). Statistical power analyses using $\mathrm{G}^{*}$ Power 3.1: tests for correlation and regression analyses. Behavior Research Methods, 41(4), 1149-60. http://doi.org/10.3758/BRM.41.4.1149

Ferguson, C. J. (2009). An effect size primer: A guide for clinicians and researchers. Professional Psychology: Research and Practice, 40(5), 532-538. http://doi.org/10.1037/a0015808

Fernández, R., Zurita, F., Linares, D., Ambros, J., Pradas de la Fuente, F., \& Linares, M. (2014). Relación entre la ansiedad estado/rasgo, posición en el terreno de juego y ocurrencia de lesiones deportivas. Universitas Psychologica, 13(2), 433-441. http://doi.org/10.11144/Javer iana.UPSY13-2.reae

Gayles, G. (2009). The student athlete experience. Design, 2009(December 2009), 65-86. http://doi.org/10.1002/ ir.311

Gayles, G., \& Hu, S. (2009). The influence of student engagment and sport participation on college outcomes among division I student athletes. The Journal of Higher Education, 80(3), 315-333. http://doi.org/10.1353/jhe.0.0051
Guardia, J. (2004). La actividad deportiva como instrumento y agente de formación académica en la Educación Superior Universitaria. Revista de Educación, 335, 95-104.

Guillén-Riquelme, A., \& Buela-Casal, G. (2011). Actualización psicométrica y funcionamiento diferencial de los items en el state trait anxiety inventory (STAI). Psicothema, 23(3), $510-515$.

Hanton, S., Neil, R., \& Mellalieu, S. D. (2008). Recent developments in competitive anxiety direction and competition stress research. International Review of Sport and Exercise Psychology, 1(790666691), 45-57. http://doi. org/10.1080/17509840701827445

Jamshidi, A., Hossien, T., Sajadi, S. S., Safari, K., \& Zare, G. (2011). The relationship between sport orientation and competitive anxiety in elite athletes. Procedia - Social and Behavioral Sciences, 30, 1161-1165. http://doi.org/10.1016/j. sbspro.2011.10.226

Jones, G. (1995). Research developments and issues in competitive anxiety in sport. British Journal of Psychology, 86, 449-478.

Jones, G., Swain, A., \& Cale, A. (1991). Gender differences in precompetition temporal patterning and antecedents of anxiety and self-confidence. Journal of Sport and Exercise Psychology, 13, 1-15.

Kerlinger, F. N. (1988). Investigación del comportamiento (2a ed.). México: McGrawHill.

Khalaila, R. (2015). The relationship between academic selfconcept, intrinsic motivation, test anxiety, and academic achievement among nursing students: Mediating and moderating effects. Nurse Education Today, 35(3), 432-438. http://doi.org/10.1016/j.nedt.2014.11.001

Koca, F. (2015). Evaluation of state and trait anxiety levels among students with no prior knowledge of skiing before and after the implementation of a skiing course. Anthropologist, 20(3), 485-491.

Landers, D., \& Boutcher, S. (1991). Relación entre arousal y la ejecución. In J. Williams (Ed.), Psicología aplicada al deporte (pp. 249-275). Madrid: Biblioteca Nueva.

Mannino, G., \& Robazza, C. (2004). Las emociones del deportista. In S. Tamorri (Ed.), Neurociencias del deporte (pp. 167-182). Barcelona: Paidotribo.

Mellalieu, S. D., Hanton, S., \& Fletcher, D. (2006). A competitive anxiety review: Recent directions in sport psychology research. New York.

Ministerio de Salud - República de Colombia. Resolución 8430, 1993 Ministerio De Salud Resolucion 1-19 (1993). Bogotá DC.

Moreno, J. A., López, M., Martínez, C., Villodre, N. A., \& Gonzallez-Cutre, D. (2007). Efeitos do gênero, a idade e a 
freqüência de prática na motivação e o desfrute do exercício físico. Fitness E Performance, 6(3), 140-146. http://doi. org/10.3900/fpj.6.3.140.p

Prieto, J. M. (2016). Corredores populares jafecta el momento de evaluación psicológica en una carrera? (In press). Rev. Guillermo de Ockham, 14(2), In print.

Roffé, M. (2009). Evaluación psicodeportológica. Buenos Aires, Argentina: Lugar.

Ruiz-Juan, F., \& Zarauz, A. (2014). Ansiedad en maratonianos en función de variables socio-demográficas Anxiety in marathoners based on socio-demographic variables. Retos. Nuevas Tendencias En Educacion Fisica, 25, 28-31.

Spielberger, C., Gorsuch, R. L., \& Lushene, R. E. (1970). The State-Trait Anxiety Inventory. Palo Alto: Consultign Phychological Press. http://doi.org/10.1037/t06496-000

Spielberger, C., \& Guerrero-Díaz, R. (2002). Inventario de ansiedad: rasgo-estado: manual e instructivo. México DF: Manual Moderno.

Von Haaren, B., Haertel, S., Stumpp, J., Hey, S., \& EbnerPriemer, U. (2015). Reduced emotional stress reactivity to a real-life academic examination stressor in students partici- pating in a 20-week aerobic exercise training: A randomised controlled trial using Ambulatory Assessment. Psychology of Sport and Exercise, 20, 67-75. http://doi.org/10.1016/j. psychsport.2015.04.004

Wadey, R., \& Hanton, S. (2008). Basic psychological skills usage and competitive anxiety responses: perceived underlying mechanisms. Research Quarterly for Exercise and Sport, 79(3), 363-373. http://doi.org/10.5641/19325030 8X13086832906030

Watt, S. K., \& Moore, J. L. (2001). Who are student athletes? New Directions for Student Services, 93, 7-18. http://doi. org/10.1002/ss. 1

Weinberg, R., \& Gould, D. (2010). Fundamentos de Psicología del deporte y del ejercicio físico ( $4^{\circ}$ ed.). Madrid: Panamericana.

Wiggins, M. S., \& Brustad, R. J. (1996). Perception of anxiety and expectations of performance. Perceptual and Motor Skills, 83, 1071-1074. http://doi.org/10.2466/ pms.1996.83.3.1071

Woodman, T., \& Hardy, L. (2003). The relative impact of cognitve anxiety and self-confidence upon sport performance: a meta-analysis. Journal of Sport Science, 21(6), 329-338. http://doi.org/10.1080/0264041031000101809 